\title{
Multiple Scenario Inversion of Reflection Seismic Prestack Data
}

\author{
Hansen, Thomas Mejer; Cordua, Knud Skou; Mosegaard, Klaus
}

Publication date:

2013

Document Version

Publisher's PDF, also known as Version of record

Link back to DTU Orbit

Citation (APA):

Hansen, T. M., Cordua, K. S., \& Mosegaard, K. (2013). Multiple Scenario Inversion of Reflection Seismic Prestack Data. Abstract from 74th EAGE Conference \& Exhibition Session, Copenhagen, Denmark.

\section{General rights}

Copyright and moral rights for the publications made accessible in the public portal are retained by the authors and/or other copyright owners and it is a condition of accessing publications that users recognise and abide by the legal requirements associated with these rights.

- Users may download and print one copy of any publication from the public portal for the purpose of private study or research.

- You may not further distribute the material or use it for any profit-making activity or commercial gain

- You may freely distribute the URL identifying the publication in the public portal

If you believe that this document breaches copyright please contact us providing details, and we will remove access to the work immediately and investigate your claim. 


\section{Copenhagen|'12}

\section{Introduction}

Inversion of prestack seismic data in a probabilistic framework is a challenging inversion problem that has been targeted in the last decades with different approaches. Buland and Omre (1993) solve a prestack inversion problem in a Bayesian context making use of normal moveout corrected prestack seismic data, and a small contrast approximation to Zoeppritz equations, that allow a linear formulation of a convolution problem to describe the relation between elastic parameters and seismic data. Using least squares inversion a Gaussian a posteriori model is computed. This a posteriori model describes the spatial variability around the maximum a posteriori model. Gouveia and Scales (1998) suggest to solve the prestack inversion problem, also in a Bayesian context, using a non-linear optimization method to find the maximum a posteriori model. The uncertainty of the model is then approximated by a local Gaussian a posteriori probability distribution centered at the maximum a posteriori model. Full waveform inversion using the adjoint approach proposed by Tarantola $(1982,1984)$, is one example of an inversion method that allow inference of elastic properties of the subsurface from prestack seismic data. This approach has proven very successful, especially in the case where transmitted waves are recorded. The outcome of this inversion is the maximum a posteriori model that minimized the data misfit. The method does not allow quantification of uncertainty of the solution, nor does the method easily take data uncertainty or existing prior information into account.

The methods described above are local methods, in that one model is found that maximizes a posteriori probability. The uncertainty, if given, is then quantified by a Gaussian probability distribution around the maximum a posteriori model. Thus, if other models exist in the space of acceptable solutions, not on the vicinity of the model with maximum a posteriori probability, then such models will not be part of the posterior uncertainty analysis, and hence this should lead to underestimation of the a posteriori uncertainty.

Here we propose a prestack inversion algorithm that will locate different models that are consistent with data, a noise model and prior information that does not depend on a smooth known background model of the elastic parameters. We will not describe the full posterior probability distribution, but try to locate models centred at point of local maximum a posteriori likelihood, consistent with data, noise and prior information. The development of the algorithm stems from practical observations dealing with manual matching of reservoir properties to seismic reflection data: In order to quantify the commercial viability of a possible reservoir identified from a reflection seismic data set, one wish to identify a number of models whose seismic response match the observed seismic data, within their uncertainties. The method we present is developed to alleviate this process.

\section{Multiple Scenario Sampling}

Tarantola and Valette (1982) formulated a probabilistic approach to solving inverse problems where all available states of information is described by probability density functions (pdfs). The solution to the inverse problem is the probability distribution obtained by combing all the known states of information. In a typical inverse problem the states of information can be described by the a priori and the likelihood pdfs. The 'a priori' pdf, $\rho_{\mathrm{M}}(\mathbf{m})$, describes the data-independent prior knowledge of the model parameters. The likelihood pdf, $L(\mathbf{m})$, is a probabilistic measure of how well the data associated to a given model, match a given model of data uncertainty. The solution to such an inverse problem is then the a posteriori pdf, $\sigma_{\mathrm{M}}(\mathbf{m})$, which is proportional to the product of the prior and the likelihood:

$$
\sigma_{M}(\mathbf{m})=k \rho_{M}(\mathbf{m}) L(\mathbf{m})
$$

The Metropolis algorithm is a Markov Chain Monte Carlo based algorithm that can be used to sample the a posteriori pdf of non-linear inverse problem in presence of complex a priori information, Mosegaard and Tarantola (1995). This algorithm is at the core of our proposed inversion algorithm 


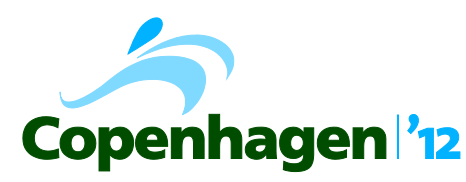

To invert prestack seismic data we propose a sampling strategy where we initially infer the low frequency Vp variations from picked reflection travel times. Then complexity of the considered data is gradually increased, in a workflow as the following:

1. Invert reflection travel time data to obtain smooth $\mathrm{Vp}$ velocity trend model. This is done by generating a sample of the a posteriori pdf using the Metropolis algorithm. This produces $\mathrm{N}$ independent realizations of the a posteriori pdf for the travel time inversion problem.

2. Invert zero offset data to infer $\mathrm{Vp}$ and density. For each of the $\mathrm{N}$ low frequent Vp models, a separate Metropolis algorithm is run. This will sample solutions around a local maximum a posteriori model.

3. Invert offset dependent prestack data to infer Vp, Vs and density. Each of the $\mathrm{N}$ Metropolis chains is continued allowing variability also of Vs

\section{Synthetic inversion of Pre stack seismic data from the Siri field in the North Sea}

Figure 1a show a well log from the North Sea, with a possible reservoir located between two way travel time depths of $1980 \mathrm{~ms}$ and $2000 \mathrm{~ms}$ (indicated by grey lines in Figure 1a). We will use this as a reference model, from which we compute a synthetic shotgather with offset from $0 \mathrm{~m}$ to $3000 \mathrm{~m}$. To compute the prestack shotgather we use a non-linear convolution model, based on ray tracing and the full Zoeppritz equations. We use a Ricker wavelet with maximum peak frequency of $25 \mathrm{~Hz}$. For the inference of the low frequent Vp velocity field we have picked the arrival times of 10 coherent reflectors. From these we have estimated a number of RMS velocities associated to a certain time depth.

Initially we consider only travel time data, and assume a priori a relatively long wavelength spatial correlation, in form of a Gaussian covariance model, with range 400 meter and standard deviation of $31 \mathrm{~m} / \mathrm{s}$. A Metropolis algorithm is run for 20000 iterations, producing an a posteriori sample of 100 realizations, as shown in Figure 1b. These clearly capture the low frequency variation of the true Vp velocity.

An independent Metropolis algorithm is started in each of 20 randomly selected smooth Vp velocity model. Initially only the zero offset waveform data is considered as data.

A priori models for the spatial variation of Vp and density are inferred from a log located near the reference log, in form of Gaussian based statistics. The prior model for Vp is described by a Spherical covariance model with range $40 \mathrm{~m}$ and a standard deviation of $200 \mathrm{~m} / \mathrm{s}$. The prior model for density is described by a Spherical covariance model with range $40 \mathrm{~m}$ and a standard deviation of $63 \mathrm{~kg} / \mathrm{m} 3$. Data noise is defined as uncorrelated Gaussian noise with a standard deviation of 0.03 , reflecting a signal to noise ratio of about 30.

In addition a correlation coefficient between $\mathrm{Vp}$ and density was found at 0.67 . Initially $\mathrm{Vp}$ and density is allowed to vary. (Vs is kept constant). After 6000 iterations all 20 running chains found a model that leads to a datafit within the considered data uncertainty. Finally, the full offset dependent seismic shotgather data set is considered as data, and both Vp, Vs and density are inferred. The prior model for the Vs is chosen as a Spherical covariance model with a range of $40 \mathrm{~m}$ and a standard deviation of $63 \mathrm{~m} / \mathrm{s}$. A correlation coefficient of 0.9 is used to describe the correlation between $\mathrm{Vp}$ and Vs. Using the final state of the 20 models from the previous run of the Metropolis algorithm another 20 algorithms is started and run for 6000 realizations. 

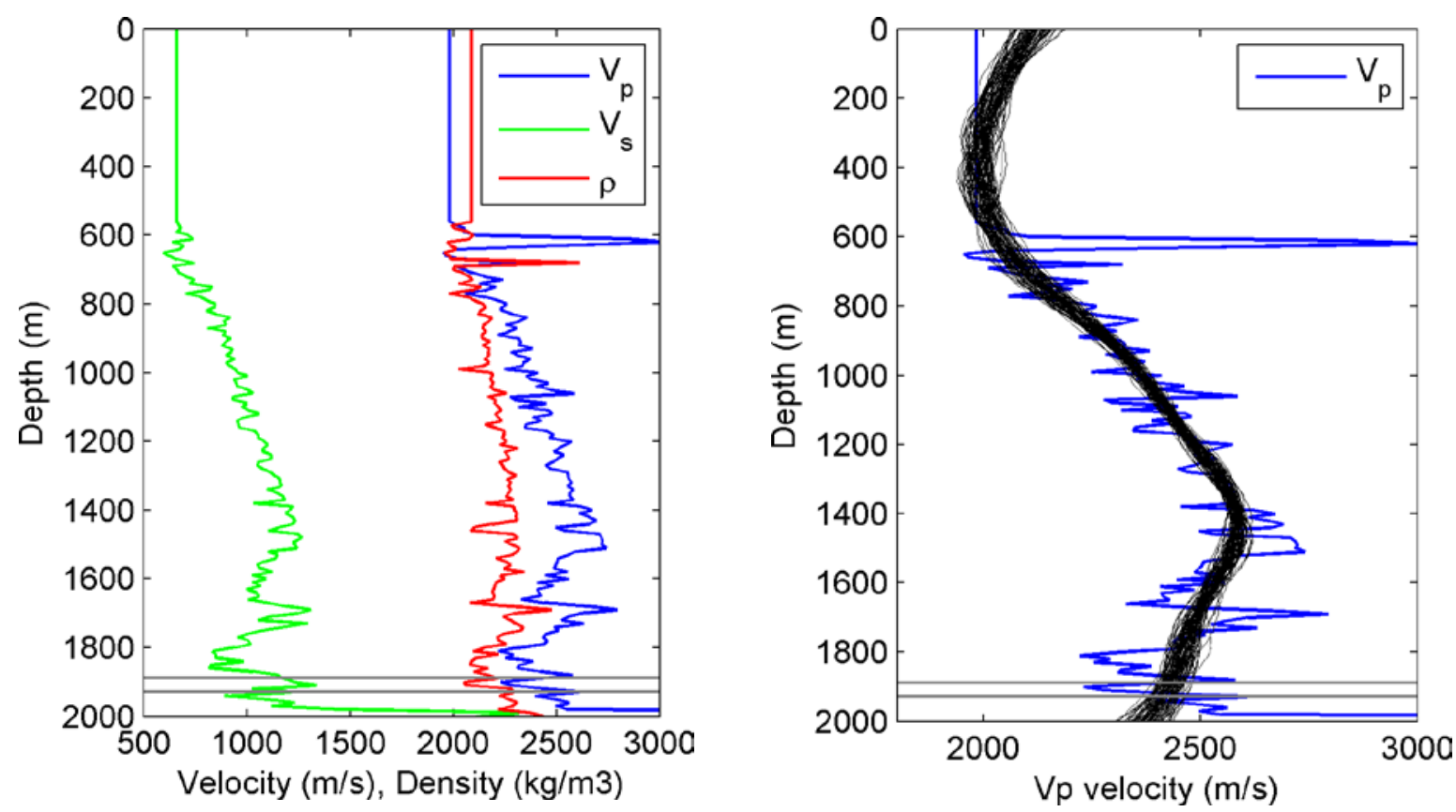

Figure 1 a,left), Reference $V p$,Vs and density log. b,right), Realizations of the a posteriori pdf, for reflection travel time inversion (black lines) on top of the reference Vp model (blue).

Figure 2 shows the final 20 realizations in a close up near the reservoir. It can be seen that all 20 models correctly identify the decrease in Vp velocity and Density within the reservoir zone. The Vs velocity however is rather poorly resolved. Figure 3a compare the observed zero offset trace (red) to the zero offset traces computed from 20 accepted models. Figure 3b compare a shotgather computed from one of the accepted models to the reference data set.

\section{Conclusions}

The main disadvantage for using a sampling algorithm as proposed is that a large number of forward calculations must be evaluated. This can become computationally unfeasible. However, the computation power keeps increasing, and some of the forward calculations considered here show promise of being very efficient using GPU enabled algorithms.

The are several benefits of using an algorithm such as the proposed. First, the method allows consideration of arbitrarily complex data noise, and very complex a priori information. Second, as the method seeks globally for local maximas it allows inference of a number of classes of models that fit the data and that honor the prior model. Third, as the the inversion relies on a raw (non-nmo corrected data set) it is sensitive to both low frequent and high frequent Vp velocity variations. Finally, the Bayesian formulation of the inversion problem allows linking between elastic properties and rock physics properties such as permeability, porosity and water saturation. In case statistical based rock physics model are available, the presented method should be able to infer for example porosity directly from seismic pre-stack data. Presently we are focusing on incorporating rock physics models to the sampling method, and extending the method to 2D problem.

\section{Acknowledgement}

We thank DONG, and specifically Niels Ter-Borch, for financial support, fruitful discussions and permission to publish these data.

\section{References}

Buland, A., and H. More, 2003, Bayesian linearized AVO inversion, Geophysics, 68 (1), 185-198. 
Gouveia, W. and J.A. Scales, 1997. Resolution of seismic waveform inversion: Bayes versus Occam: Inverse Problems, 13, 323-349.

Mosegaard, K., and A. Tarantola, 1995, Monte Carlo sampling of solutions to inverse problems, Journal of Geophysical Research, 100 (B7), 12431-12447.

Tarantola, A., and B. Valette, 1982, Inverse Problems =

Quest for Information: Journal of Geophysics., 50, 159-170.

Tarantola, A., 1986, A strategy for nonlinear elastic inversion of seismic reflection data, Geophysics, 51, 1893-1903.

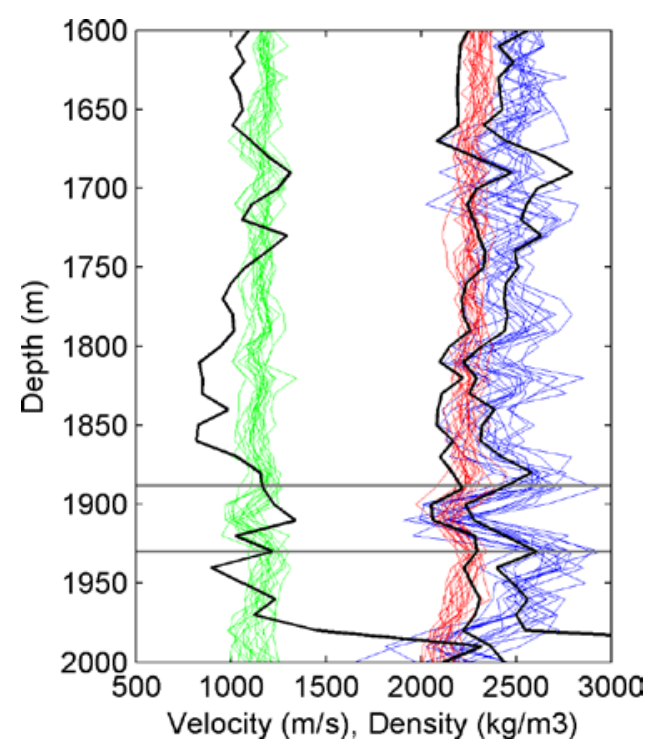

Figure 220 models that result in a datafit that match observed data within its uncertainty.
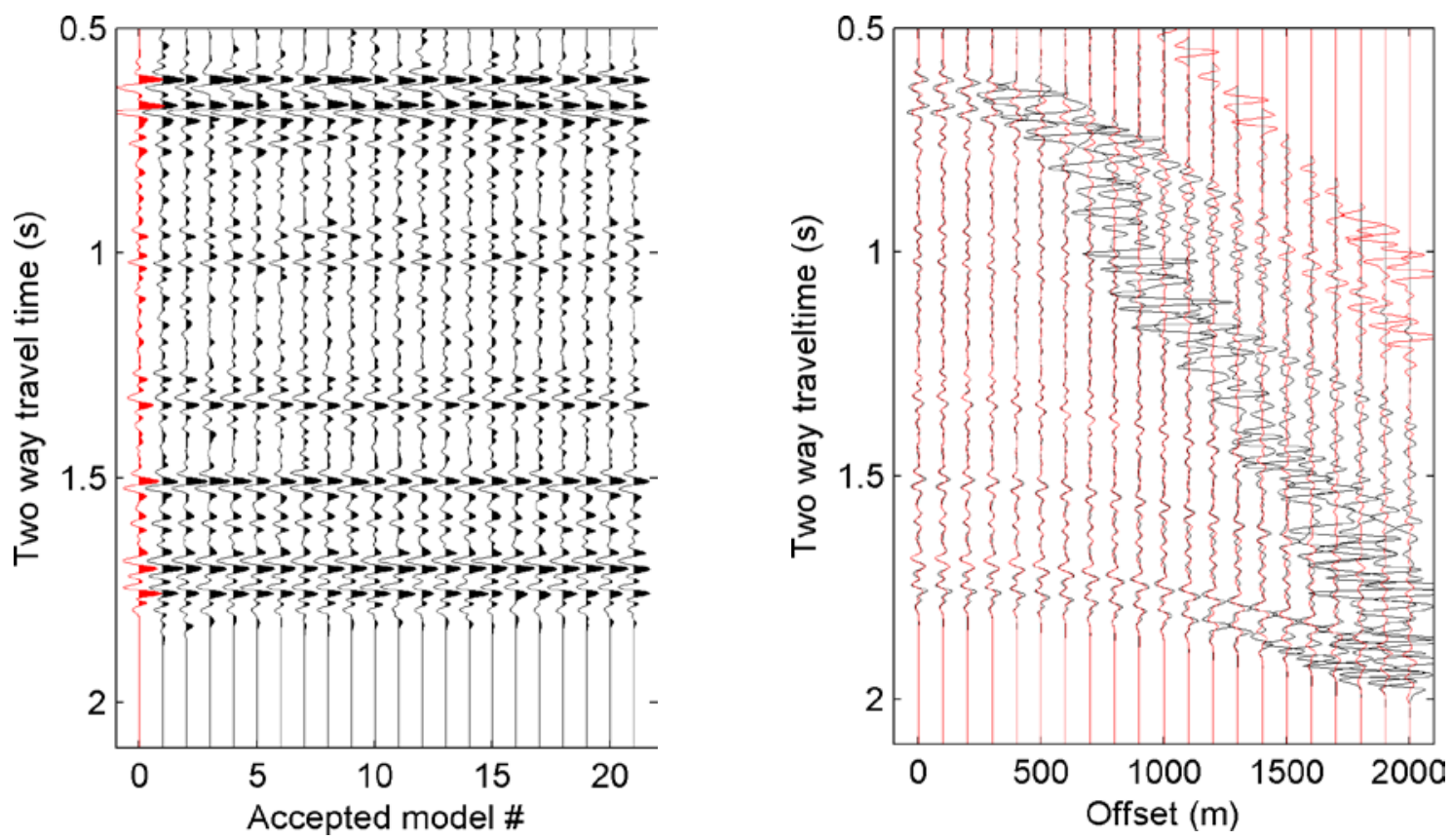

Figure 3 a, left) Zero offset seismic response from 21 accepted models. The first (red) trace is the zero offset reference trace. b,right) Comparison of the reference shotgather data (black lines) and an (typical) example of the data response from one of the 20 accepted models, of Figure 2 